\title{
Ultrafast TEM and EELS Based on Microwave Cavities
}

Jom Luiten

Eindhoven University of Technology, Eindhoven, Noord-Brabant, Netherlands

Ultrafast Transmission Electron Microscopy (U-TEM) has become a very important tool for the study of ultrafast phenomena at (sub-)nm length scales and (sub-)ps time scales. U-TEM is usually based on the creation of ultrashort electron pulses by femtosecond laser photoemission from a flat cathode, with the result that both the beam quality and the average current are significantly less than in state-of-the-art continuous-beam TEMs. At Eindhoven University we have developed U-TEM in which ultrashort electron pulses are produced by using a $3 \mathrm{GHz}$ deflecting cavity in $\mathrm{TM}_{110}$ mode, synchronized to a femtosecond laser, to sweep a high-brightnes continuous beam across a slit. In this way the beam quality and the sub-eV energy spread of the FEG source of an adapted $200 \mathrm{keV}$ Tecnai TEM are conserved, enabling near-atomic spatial resolution with sub-ps temporal resolution [1]. First results of pump-probe experiments will be presented.

In addition we have developed a new method for doing Time-of-Flight Electron Energy Loss Spectroscopy (ToF-EELS) based on the combined use of two TM110 deflecting cavities and two TM010 (de)compression cavities. The first 'chopping' TM110 cavity produces ultrashort electron pulses which are sent through a sample. Energy loss in the sample translates into reduction of the electron velocity and thus into a later arrival time at the detector, which is measured with a synchronized second TM110 'streak' cavity. In this way an energy resolution of $12 \mathrm{eV}$ at $30 \mathrm{keV}$ has been demonstrated [2]. By adding a TM010 cavity in compression mode after the sample, the longitudinal phase space can be manipulated in such a way that the energy resolution is improved to $2 \mathrm{eV}$ [3]. By adding a second $\mathrm{TM}_{010}$ cavity in stretching mode before the sample, the electron pulses can be monochromated without loss of current. Detailed charged particle tracking simulations show that an energy resolution of $20 \mathrm{meV}$ combined with a temporal resolution of 2 ps can then be achieved. The 4-cavity ToF-EELS setup is currently under construction and first results will presented.

\section{References}

[1] W. Verhoeven, J.F.M. van Rens, E.R. Kieft, P.H.A. Mutsaers, and O.J. Luiten, High quality ultrafast transmission electron microscopy using resonant microwave cavities, Ultramicroscopy 188, 85 (2018) [2] W. Verhoeven, J.F.M. van Rens, M.A.W. van Ninhuijs, W.F. Toonen, E.R. Kieft, P.H.A. Mutsaers, and O.J. Luiten, Time-of-flight electron energy loss spectroscopy using TM110 deflection cavities, Struct. Dyn. 3, 054303 (2016)

[3] W. Verhoeven, J.F.M. van Rens, W.F. Toonen, E.R. Kieft, P.H.A. Mutsaers, and O.J. Luiten, Timeof-flight electron energy loss spectroscopy by longitudinal phase space manipulation with microwave cavities, Struct. Dyn. 5, 051101 (2018) 